\title{
Variational analysis of driven-dissipative Rydberg gases
}

\author{
Hendrik Weimen* \\ Institut für Theoretische Physik, Leibniz Universität Hannover, Appelstr. 2, 30167 Hannover, Germany
}

(Dated: July 25, 2018)

\begin{abstract}
We study the non-equilibrium steady state arising from the interplay between coherent and dissipative dynamics in strongly interacting Rydberg gases using a recently introduced variational method [H. Weimer, Phys. Rev. Lett. 114, 040402 (2015)]. We give a detailed discussion of the properties of this novel approach, and we provide a comparison with methods related to the Bogoliubov-Born-Green-Kirkwood-Yvon hierarchy. We find that the variational approach offers some intrinsic advantages, and we also show that it is able to explain the experimental results obtained in an ultracold Rydberg gas on an unprecedented quantitative level.
\end{abstract}

PACS numbers: 32.80.Ee, 03.65.Yz, 05.30.Rt

\section{INTRODUCTION}

The discovery of dissipative quantum state engineering [1 3], i.e., the use of controlled sources of dissipation for the preparation of many-particle quantum states, has led to a surge of interest in open quantum many-body systems. The unrivaled tunability of interaction and dissipation properties of driven Rydberg gases makes them particularly useful for this purpose and has resulted in a large amount of theoretical and experimental works investigating the interplay between coherent and dissipative dynamics in these systems [4-24]. Here, we provide a detailed discussion of the application of a recently introduced variational principle for the steady state of dissipative quantum many-body systems [25] to drivendissipative Rydberg gases.

Rydberg atoms are routinely excited from ground state atoms by coherent laser driving, with their atomic properties and interaction strength scaling dramatically with the principal quantum number [26]. The radiative decay of the metastable Rydberg state provides a natural dissipative element whose decay rate can be widely tuned by laser coupling to other excited non-Rydberg states [27]. As such, Rydberg atoms provide an ideal environment for studying dissipative many-body dynamics in a strongly interacting regime.

In this article, we perform an analysis of the nonequilibrium steady state of a driven-dissipative Rydberg gas. We investigate the properties of this steady state using a variational method recently introduced by the author [25]. We provide a detailed evaluation of the approximations carried out within this novel method. We complement our analysis of the variational method by describing an alternative approach to the investigation of dissipative quantum many-body systems based on a hierarchy of equation that also allows for a systematic incorporation of correlations. We perform an explicit comparison of the two methods, finding substantial advantages in favor of the variational approach. Finally,

*Electronic address: hweimer@itp.uni-hannover.de we demonstrate that the variational method provides remarkable quantitative agreement with the experimental results on the steady state phase transition observed in an ultracold Rydberg gas.

\section{DRIVEN-DISSIPATIVE RYDBERG GASES}

We first give a microscopic description of drivendissipative Rydberg gases. We express the dynamics in terms of a spin 1/2 representation, where the down spin state corresponds to an electronic ground state, and the up spin state refers to a highly excited Rydberg state [26]. As the frequency difference between all electronic states is much larger than the decay rate of the Rydberg state, it is well justified to describe the radiative decay of the Rydberg excitations in terms of a Markovian quantum master equation in Lindblad form,

$$
\frac{d}{d t} \rho=-\frac{i}{\hbar}[H, \rho]+\sum_{i}\left(c_{i} \rho c_{i}^{\dagger}-\frac{1}{2}\left\{c_{i}^{\dagger} c_{i}, \rho\right\}\right)
$$

where $\rho$ is the density operator describing the state of the system, $H$ is the Hamiltonian accounting for the coherent part of the dynamics, and the set of $c_{i}$ are quantum jump operators responsible for the dissipation [28]. If the external laser fields are close to a resonance between the atomic ground state and a single Rydberg state, such a system in its rotating frame is governed by the spin $1 / 2$ Hamiltonian

$$
H=-\frac{\hbar \Delta}{2} \sum_{i} \sigma_{i}^{z}+\frac{\hbar \Omega}{2} \sum_{i} \sigma_{i}^{x}+\sum_{i<j} \frac{C_{6}}{\left|\mathbf{r}_{i}-\mathbf{r}_{j}\right|^{6}} P_{i}^{r} P_{j}^{r}
$$

where the Rabi frequency $\Omega$ and the detuning $\Delta$ represent the laser parameters, the $C_{6}$ coefficient denotes the strength of the van der Waals repulsion between Rydberg states, and $P_{i}^{r}=\left(1+\sigma_{i}^{z}\right) / 2$ is the projection onto the Rydberg state. The jump operators describing the decay of the Rydberg excitations is represented by quantum jump operators describing up spins flipping into down spins according to $c_{i}=\sqrt{\gamma} \sigma_{-}^{(i)}$, with $\gamma$ being the decay rate of the Rydberg state. Initially, we will assume that 
the system is well described by a model involving only nearest-neighbor interactions, i.e., the blockade radius is smaller than the lattice spacing $a$. Then, we can introduce an interaction constant given by $V=C_{6} / a^{6}$. Of special interest is the regime where the Hamiltonian is equivalent to a transverse field Ising model, which is realized for $\Delta=V / 2$.

In contrast to closed quantum systems, an open quantum systems such as the dissipative Rydberg gas will generically relax towards a stationary state characterized by the condition $\frac{d}{d t} \rho=0$. Crucially, the interplay between coherent and incoherent part of the dynamics generically leads to a stationary state that is different from any state in thermal equilibrium, i.e., a nonequilibrium steady state.

\section{VARIATIONAL PRINCIPLE}

In the following, we review the basic concepts behind the variational principle introduced in Ref. [25]. The basic idea is to take a variational trial state $\rho$ and compute the residual dynamics it will generate by computing its time-derivative $\dot{\rho}=\mathcal{L} \rho$ according to the underlying quantum master equation with the Liouvillian superoperator $\mathcal{L}$. The operator $\dot{\rho}$ can be expressed as a traceless Hermitian matrix. To find the variational approximation to the true steady state having $\dot{\rho}=0$, we choose the variational state that minimizes the trace norm of $\dot{\rho}$, i.e.,

$$
\rho_{\text {var }}=\underset{\rho}{\arg \min } \operatorname{Tr}\{|\mathcal{L} \rho|\} .
$$

Choosing the trace norm as the correct matrix norm can be motivated on two different grounds. First, the trace norm is unbiased in the sense that it does not favor certain classes of variational states over others without a physical reason. This is related to the linearity condition $\|\dot{\rho}\|=\|\lambda \dot{\rho}\| / \lambda$ satisfied by the trace norm. In particular, any other Schatten norm $\|\dot{\rho}\|=\operatorname{Tr}\left\{|\dot{\rho}|^{p}\right\}$ with $p>1$ is biased towards the maximally mixed state [25].

The second way to motivate the choice of the trace norm follows follows from quantum information theory. Here, the trace norm can be interpreted as being equivalent to the trace distance of $\dot{\rho}$ to the zero matrix, while the latter is obtained for $\dot{\rho}$ if and only if the variational state $\rho$ is an exact stationary state of the master equation. Importantly, the ability to physically distinguish the operator $\dot{\rho}$ from the zero matrix is given by their trace distance [29], and hence the trace norm of $\dot{\rho}$. In this sense, the trace norm is the natural norm to decide which variational state is the best approximation to the true steady state. We would also like to point out that the trace norm for the steady state is equivalent to applying a time-dependent variational principle for the dynamical evolution [30].

Following these initial statements, we can now proceed with the variational analysis. Calculating the trace norm is in general still a computational problem scaling exponentially with the system size, so additional steps are needed first. This situation is very similar to that of correlated fermions, where energy expectation values for variational Gutzwiller wave functions can only be evaluated within further approximations [31]. In our case, we exploit the fact that we are not so much interested in the actual value of the trace norm, but rather in the properties of the stationary state. The additional approximations we will carry out retain the variational character of our calculation, i.e., they provide a rigorous upper bound to the trace norm.

\section{A. Product states}

To be explicit, we first consider the case of the variational set of states being restricted to product states, i.e.,

$$
\rho=\mathcal{R} 1=\prod_{i} \rho_{i} .
$$

Here, we have introduced the superoperator $\mathcal{R}$, which replaces every occurrence of the identity operator for site $i, 1_{i}$, by the single-site density matrix $\rho_{i}$. Additionally, we focus on quantum master equations including nearest-neighbor interactions or jump operators involving at most two adjacent sites. Then, the trace norm of the resulting dynamics can be written in the form

$$
\|\dot{\rho}\|=\left\|\sum_{i} \mathcal{R} \dot{\rho}_{i}+\sum_{\langle i j\rangle} \mathcal{R} \dot{C}_{i j}\right\|,
$$

where $\dot{\rho}_{i}=\operatorname{Tr}_{i}\{\dot{\rho}\}$ describes the single-site dynamics and $C_{i j}$ accounts for correlations between the sites. Here, the correlations between the sites stem from the nearestneighbor interactions and two-site jump operators and thus are restricted to nearest-neighbor correlations only. As a first approximation, we apply the triangle inequality to pull the summation over $i$ out of the norm,

$$
\|\dot{\rho}\| \leq \sum_{i}\left\|\mathcal{R} \dot{\rho}_{i}+\sum_{j} \mathcal{R} \dot{C}_{i j}\right\| .
$$

As the next step, we make use of the fact that $\dot{\rho}_{i}$ and $\dot{\rho}_{j}$ act on different parts of the Hilbert space, which allows us to write

$$
\|\dot{\rho}\| \leq \sum_{i}\left\|\mathcal{R} \dot{\rho}_{i}+\sum_{j} \mathcal{R}\left(\rho_{i} \dot{\rho}_{j}+\dot{C}_{i j}\right)\right\| .
$$

Note that this inequality does not change the variational approximation to the steady state, but it allows us to write the final result in a more compact form. Finally, we employ the triangle inequality a second time, yielding

$$
\|\dot{\rho}\| \leq \sum_{\langle i j\rangle}\left\|\mathcal{R}\left(\dot{\rho}_{i} \rho_{j}+\rho_{i} \dot{\rho}_{j}+\dot{C}_{i j}\right)\right\|=\sum_{\langle i j\rangle} \operatorname{Tr}\left\{\left|\dot{\rho}_{i j}\right|\right\}
$$

Consequently, we have succeeded in mapping the full quantum many-body problem into a sum of efficiently 


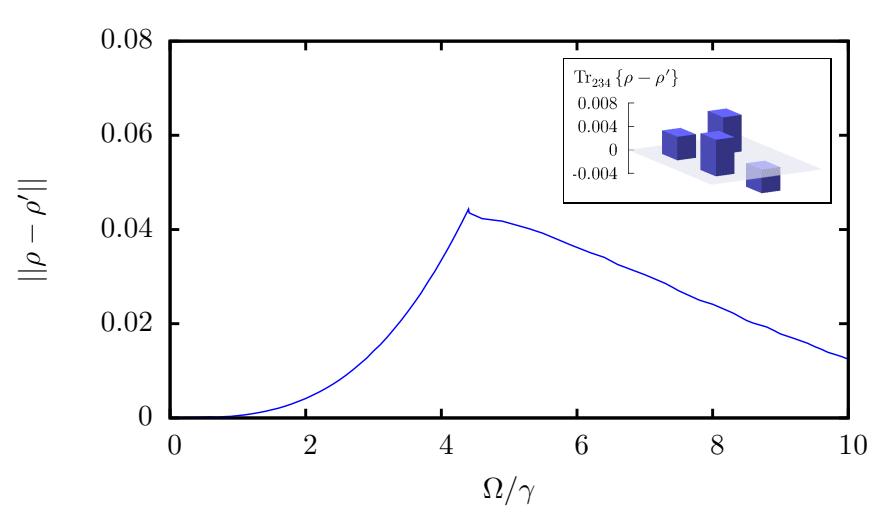

FIG. 1: Difference in the variational states introduced by the inequalities leading to Eq. (8) as indicated by the trace distance between the states $\rho$ and $\rho^{\prime}$. Here the full space density matrix $\rho$ was taken as a product state of four sites. The inset shows the difference in the single particle reduced density matrices at $\Omega=4 \gamma(\Delta=V / 2, V=5 \gamma)$.

solvable problems involving only neighboring sites. For translationally invariant systems it is in general even sufficient to solve a single two-site problem.

At this point, it becomes a natural question to ask how well justified our approximations are. Besides the obvious approximation in restricting to the variational manifold, we have to assess the deviations introduced by the inequalities leading to Eq. (8). For small system sizes, we can answer this question exactly because the Hilbert space is still small enough so that we can minimize the trace norm of Eq. (3) without any additional approximations. As shown in Fig. 1, the deviation introduced by the inequalities is quite small for the Ising model describing the dissipative Rydberg gas and in the case of four particles.

Of course, we are not really interested in problems involving four sites, but rather see how the situation behaves in the thermodynamic limit. For this, we can check how the exact variational norm behaves as a function of system size. As a single evaluation of the norm is computationally less costly than performing a full minimization, we can go to somewhat larger system sizes. Remarkably, we find that the scaling with system size is completely independent of the model being investigated or the trial state for which the norm is evaluated, see Fig. 2 For a simple non-interacting toy model, this surprising fact can be understood as a consequence of the central limit theorem. This model consists of $N$ purely dissipative two-level systems, whose jump operators are given by a dissipative spin-flip of the form $c_{i}=\sqrt{\gamma} \sigma_{i}^{-}$, as in the case of the dissipative Rydberg gas. As a trial state, we choose the maximally mixed state, $\rho=1 / 2^{N}$. Since the model is purely classical and non-interacting, we can give an analytical expression for the trace norm of the master

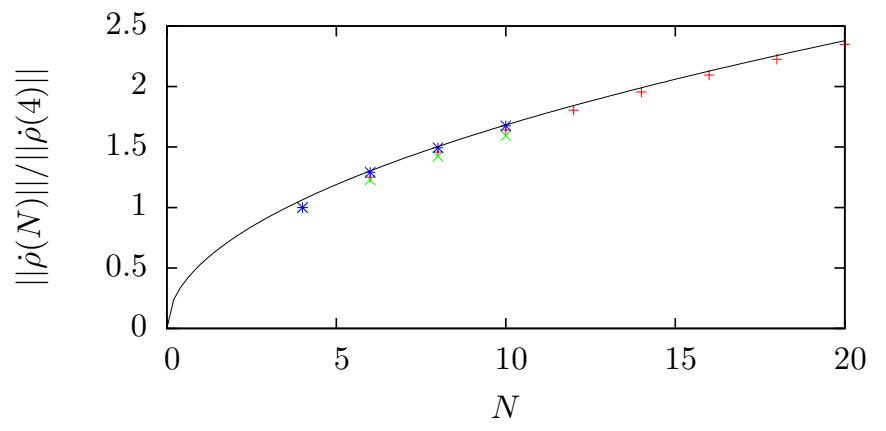

FIG. 2: Trace norm of the master equation depending on the system size $N$ for different models and different product states normalized to the value at $N=4$. The asymptotic behavior for the noninteracting decay model is shown as a solid line.

equation,

$$
\|\dot{\rho}\|=4 \sum_{m=0}^{N / 2}\left(\begin{array}{c}
N \\
\frac{N}{2}-m
\end{array}\right) \frac{m}{2^{N}} \gamma .
$$

This expression can be evaluated efficiently even for large values of $N$ and corresponds to the crosses in Fig. 2 To obtain the asymptotic behavior in the limit of large $N$, we replace the sum by an integral and use the central limit theorem to approximate the binomial coefficients by a Gaussian function, obtaining

$$
\begin{aligned}
\|\dot{\rho}\| & \approx \int_{0}^{N / 2} \frac{4}{\sqrt{\pi N / 2}} \exp \left[-\frac{\left(x-\frac{N}{2}\right)^{2}}{\frac{N}{2}}\right]\left(\frac{N}{2}-x\right) \gamma d x \\
& =\sqrt{\frac{2 N}{\pi}} \gamma+O(\sqrt{N \exp [-N]}) .
\end{aligned}
$$

As shown in Fig. 2, this asymptotic behavior is already reached for quite small values of $N$, indicating that the central limit theorem can also be applied to the fully quantum case, which appears to be a natural consequence of the correlations in $\dot{\rho}$ being restricted to nearest neighbors. Therefore, we conclude that the approximations needed for an efficient calculation of the variational norm are well justified and enable to use the variational principle as a powerful tool to compute steady state properties.

\section{B. Correlations}

The considerations made for product states can also be extended towards more generic classes of variational states including correlations. Here, we study the case where nearest-neighbor correlations are fully included, resulting in a variational state according to

$$
\rho=\prod_{i} \rho_{i}+\sum_{\langle i j\rangle} \mathcal{R} C_{i j}+\sum_{\langle i j\rangle \neq\langle k l\rangle} \mathcal{R} C_{i j} C_{k l}+\ldots
$$

Additionally, we impose the constraint that all reduced density matrices $\rho_{i j}=\rho_{i} \rho_{j}+C_{i j}$ are positive definite. 
Using the same steps as for product states and observing that partial traces of correlations vanish, $\operatorname{Tr}_{i}\left\{C_{i j}\right\}=0$, we can again find an upper bound to the variational norm as

$$
\|\dot{\rho}\| \leq \sum_{\langle i j k\rangle}\left\|\dot{\rho}_{i j k}\right\|
$$

Here, the many-body problem reduces to a sum of threesite problems. It should not be surprising that the inclusion of all two-site correlations leads to the minimization of a three-site problem as the interaction generically generates one higher order of correlations. Consequently, treating $n$-body correlations exactly requires to solve an $n+1$-body minimization problem.

\section{HIERARCHY EQUATION METHODS}

An alternative way to analyze dissipative manybody dynamics is through a hierarchy of equations in terms of their correlations, in close analogy to the Bogoliubov-Born-Green-Kirkwood-Yvon hierarchy of classical physics [32]. For this, we express the density operator $\rho$ in terms of their reduced density matrices, according to the generating functional

$$
\mathcal{F}(\alpha)=\log \operatorname{Tr}\left\{\rho \prod_{i}\left(1_{i}+\alpha_{i}\right)\right\},
$$

where the operators $\alpha_{i}$ form an arbitrary operator basis acting on lattice site $i$ 33]. The first terms of the hierarchy are then given by

$$
\begin{aligned}
\rho_{i} & =\operatorname{Tr}_{i j}\{\rho\}=\left.\frac{\partial \mathcal{F}}{\partial \alpha_{i}}\right|_{\alpha=0} \\
\rho_{i j} & =\operatorname{Tr}_{i j j}\{\rho\}=\rho_{i} \rho_{j}+\underbrace{\left.\frac{\partial^{2} \mathcal{F}}{\partial \alpha_{i} \partial \alpha_{j}}\right|_{\alpha=0}}_{C_{i j}} \\
\rho_{i j k} & =\operatorname{Tr}_{i j j k}\{\rho\}=\rho_{i} \rho_{j} \rho_{k}+C_{i j} \rho_{k}+C_{i k} \rho_{j}+C_{j k} \rho_{i} \\
& +\left.\frac{\partial^{3} \mathcal{F}}{\partial \alpha_{i} \partial \alpha_{j} \partial \alpha_{k}}\right|_{\alpha=0} .
\end{aligned}
$$

The same analysis can also be performed on the level of the quantum master equation to obtain effective equations of motion for the reduced density matrices. Generically, each equation of motion is coupled to the next higher equation of motion in the hierarchy. The usual strategy is then to truncate the hierarchy at some point by setting the contributions from all higher order derivatives of $\mathcal{F}$ to zero and solve the resulting closed set of equations [33]. This approximation is attributed to a $1 / z$ suppression of the higher order derivatives. In lowest order, the resulting equations of motion are identical to the mean-field decoupling of Ref. 34].

In principle, it is possible to systematically incorporate correlations similar as in the variational approach by going up to higher terms in the hierarchy. However, the main drawback of the method remains, that it cannot be formulated in terms of a variational principle, i.e., the neglected higher order terms are uncontrolled. In the case of the hierarchy equations having multiple solutions, it is actually possible to combine it with the variational method. First, all solutions to the hierarchy equations are computed, which are then used as a variational class of states to find the solution that leads to a minimization of the variational norm.

\section{RESULTS}

\section{A. Lattice model}

We will first put our attention to the case where the atoms are distributed on a two-dimensional square lattice. We will make a direct comparison between the variational method and the results from solving the hierarchy equations. We complement this comparison with results from a numerical solution of the quantum master equation using a quantum trajectories method [35]. To ensure a comparison on an equal footing, we will compare the variational results for product states to the first order hierarchy equations, and the variational method for correlated states to the second order hierarchy equations. In the latter case, we include only nearest-neighbor correlations within both methods. The results are shown in Fig. 3. Except for intermediate values of $\Omega$, the two methods agree very well, and for correlated states, the level of agreement is further improved and also matches well with the results from the quantum trajectories simulation.

However, some important qualitative differences remain even when correlations are included. The bistability of the first order hierarchy solution is still present, although over a smaller range of parameters. Consequently, this bistability is not just an artifact of the first order result and the approximation of neglecting the $1 / z$ corrections to it. Rather, it appears to be a generic element of the hierarchy equation method. In contrast, the variational solution always produces a unique stationary state, as even in the case of multiple local minima of the variational norm, there is always a unique global minimum. Within the variational approach, we find a first-order phase transition between a low-density gas of Rydberg excitations and a high-density liquid [25].

Finally, we turn to the parameter regime for nonzero detuning $\Delta$ where the mean-field solution (i.e, the firstorder hierarchy equation) predicts the existence of an antiferromagnetic phase [5]. Within the variational method, we also find such an antiferromagnetic phase, see Fig. 4, but its extension is reduced significantly. 

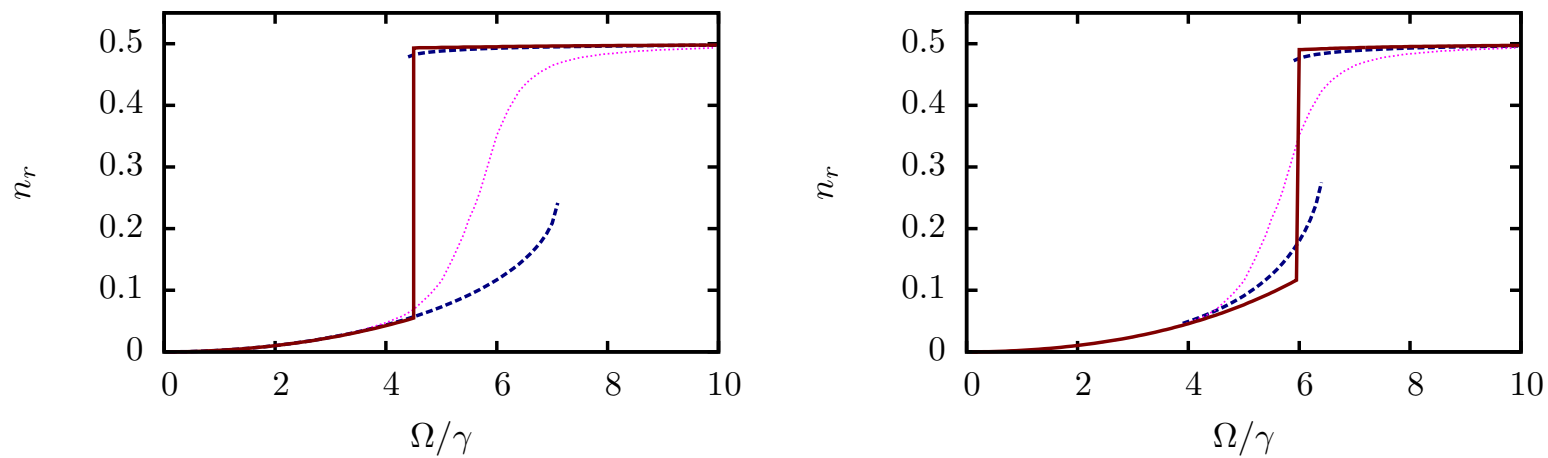

FIG. 3: Comparison of the Rydberg density $n_{r}$ for the variational solution (solid) and the solutions of the hierarchy equations (dashed) for product states (left) and correlated states (right). For reference, the quantum trajectories solution of the quantum master equation for a $4 \times 4$ system is shown as a dotted line. The data for both the variational and quantum trajectories solutions is taken from Ref. [25] $(\Delta=V / 2, V=5 \gamma)$.

\section{B. Infinite dimension limit}

The qualitative differences between the variational approach and the hierarchy equation method warrants further discussion, especially regarding the appearance of the bistable region. Some previous works have interpreted this region as a genuine thermodynamic phase [5, 21]. In this context, the concept of the lower critical dimension is particularly important. It refers to the spatial dimension of a system above which a phase transition can be observed [36]. For example, the equilibrium liquid-gas transition belongs to the Ising universality class and has a lower critical dimension of one.

In the following, we will investigate the dissipative Rydberg gas in the limit where the coordination number $z$ goes to infinity. In this case both the variational method and the first-order hierarchy equations become
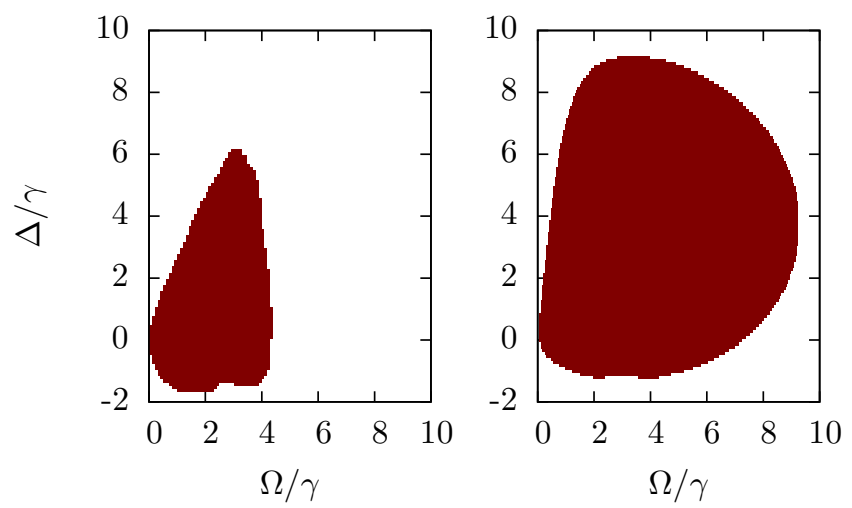

FIG. 4: Extension of the antiferromagnetic phase. The shaded area depicts the presence of antiferromagnetic order according to the variational approach (left) and the first-order hierarchy equations (right). $(V=5 \gamma)$ exact, as the true steady state of the master equation will be given by a product state. In particular, it is instructive to look at this limit to investigate the role of the bistability found in the solution of the hierarchy equations. For this, we analyze the residual dissipation between the two local minima found by the variational method. As shown in Fig. 5, the variational method always yields a unique steady state. Here we find that the larger the coordination number becomes, the smaller the difference in residual dissipation. However, the regime of true bistability indicated by a vanishing of the slope of the variational norm is only reached asymptotically as $1 / z$, and for any finite value of $z$ (i.e., for finite spatial dimensionality), there is no bistability. Consequently, it is incorrect to interpret the bistable behavior predicted by the hierarchy equations as a signature of a genuine thermodynamic phase, as it does not have a finite lower critical dimension.

Rather, these findings suggest that the variational method is the correct starting point from which arguments in favor of the existence of thermodynamic phases in sufficiently high dimensions can be based. This is of course in contrast with equilibrium systems, where such arguments can be made based on a mean-field decoupling, which is the equivalent of the first-order hierarchy equations.

It is worth mentioning that the situation is very different when the hierarchy equations predict an antiferromagnetic phase. There, we also find an antiferromagnetic phase within the variational method, albeit with a smaller extension. While these results puts the existence of such an ordered phase on firmer grounds, the role of quantum fluctuation could still preclude its observation in actual experiments, if the lower critical dimension of the transition is three or larger. 

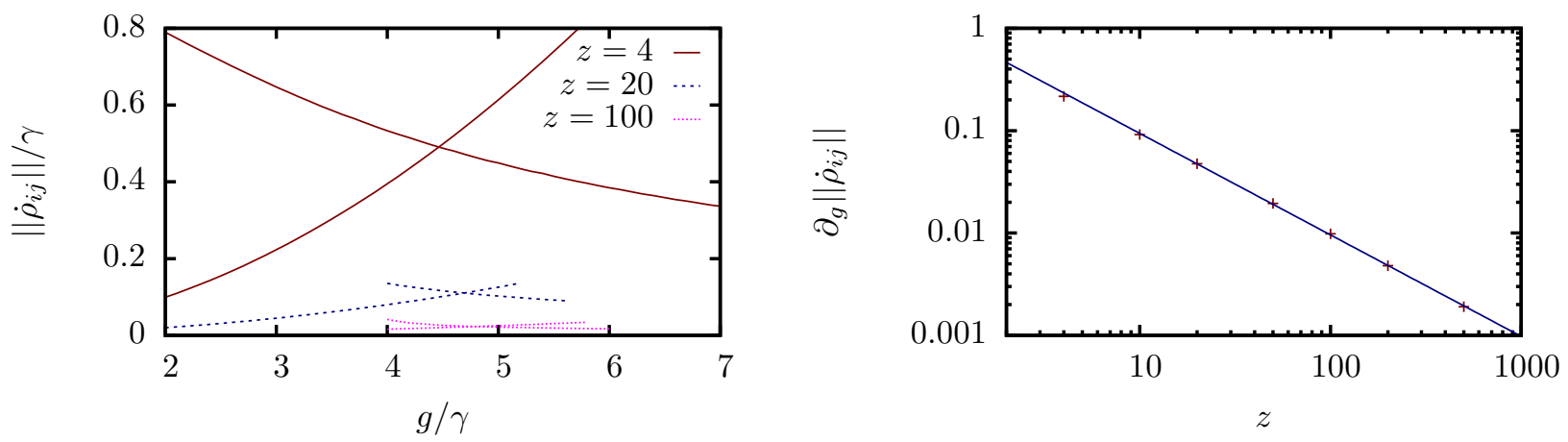

FIG. 5: Analysis of the dissipative Rydberg gas in the limit of large coordination number $z$. The left panel shows the position of the first-order transition indicated by the crossing of the two local minima in the variational norm. The right panel displays the slope of the variational norm right below the transition point. The solid line is an algebraic fit to the data with an exponent of $-0.99 \pm 0.01$, perfectly consistent with a $1 / z$ behavior.

\section{Superatom model}

We now extend the previous discussion of the lattice model of Sec. $\nabla \mathrm{TA}$ to the case of a continuum, as it has been the case in the experimental situation in Ref. [19]. In such a case, the Rydberg blockade will ensure that Rydberg excitations spontaneously form ordered structures [37, 38]. Although these correlations are shortranged, we may still well replace the underlying continuum by a lattice structure with a lattice spacing corresponding to the typical spacing between Rydberg excitations. We can then determine the lattice spacing in a self-consistent manner [39], finding

$$
z \frac{C_{6}}{a^{6}}=\sqrt{\Omega_{\mathrm{eff}}^{2}+(2 \Delta)^{2}}
$$

The factor of two in front of the detuning $\Delta$ can be understood as realizing the antiblockade condition $C_{6} / a^{6}=2 \Delta$ in the limit of vanishing Rabi frequency. The effective Rabi frequency $\Omega_{\text {eff }}$ is derived from a renormalization of the atomic Rabi frequency $\Omega$ due to a (limited) collective enhancement. Far away from resonance, the transition to the first Rydberg excitation is still collectively enhanced, but the second Rydberg excitation can then only appear at specific positions that satisfy the antiblockade condition. Assuming there is always exactly one distance where the antiblockade condition is fulfilled, we find that we can describe the dynamics of such a superatom in terms of the number of atoms inside the superatom, $N_{s}$, by renormalizing the Rabi frequency as $\Omega_{\mathrm{eff}}=N_{s}^{1 / 4} \Omega$, i.e., the geometric mean of the Rabi frequency for the first and the second Rydberg excitation.

In the following, we assume the underlying lattice to be a cubic lattice (i.e, $z=6$ ), however, we would like to stress that our results are basically independent of $z$, as long as it chosen consistently with the assumed lattice structure. Then, we can compute the number of atoms per superatom to be

$$
N_{s}=n \sqrt{\frac{C_{6}}{2 \sqrt{\Delta^{2}+\Omega^{2}}}},
$$

with $n$ being the density of ground state atoms. In the experimental situation of Ref. [19], the system was either on resonance or far away from it, i.e., either the condition $\Omega \gg \Delta$ or $\Omega \ll \Delta$ has been fulfilled. Additionally, we capture the experimental situation by including a dephasing term associated with the laser linewidth $\kappa$, according to the jump operators $c_{i}^{\prime}=\sqrt{2 \kappa} P_{r}^{(i)}$ [40].

We are now in the position to compute the steady state of the system using the variational approach for correlated states. Remarkably, our results are in good quan-
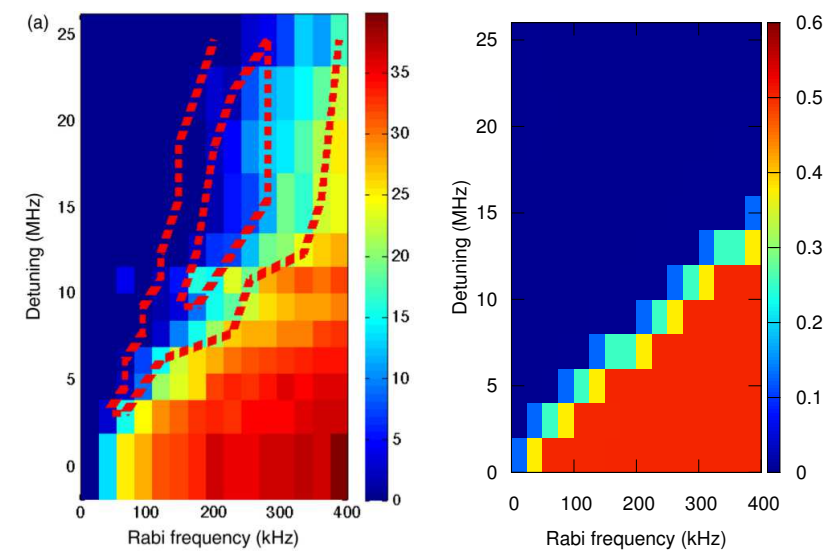

FIG. 6: Comparison of the stationary state of a dissipative Rydberg gas obtained by the Pisa experiment (left, taken with permission from [19]) and by the variational method (right). For the experimental data, the color coding refers to the total number of Rydberg excitations in the sample, while for the numerical simulations, it represents the fraction of excited superatoms $\left(\gamma=5 \mathrm{kHz}, \kappa=500 \mathrm{kHz}, n=1.8 \times 10^{17} \mathrm{~cm}^{-3}\right.$, $\left.C_{6}=7.54 \times 10^{-58} \mathrm{Jm}^{-6}\right)$. 
titative agreement with the experimental observations in Ref. [19], see Fig. 6. The jump in the density of Rydberg excitations corresponds to the first-order phase transition between a low-density gas and a high-density liquid, see Sec. $\mathrm{VA}$. In close analogy to the classical liquid-gas transitions, the first-order transition ends in a critical point. However, due to experimental limitations on the dephasing rate and on the Rabi frequency, it appears that the observation of the critical region of the dissipative Rydberg gas remains a significant challenge.

\section{SUMMARY}

In summary, we have given are detailed discussion of the recently introduced variational principle for steady states of dissipative quantum many-body systems [25]. We have exemplified its usefulness by focusing on the driven-dissipative Rydberg gas, and we have made a comparison of the variational approach to hierarchy equation methods, finding severe conceptual advantages in favor of the variational approach. Finally, we have found remarkable quantitative agreement with experimental data for the phase transition between a low-density gas of Rydberg excitations and a high-density liquid. Our results strengthen the position of the variational method as a key tool to analyze dissipative quantum many-body systems.
[1] S. Diehl, A. Micheli, A. Kantian, B. Kraus, H. P. Büchler, and P. Zoller, Quantum states and phases in driven open quantum systems with cold atoms, Nature Phys. 4, 878 (2008).

[2] F. Verstraete, M. M. Wolf, and J. Ignacio Cirac, Quantum computation and quantum-state engineering driven by dissipation, Nature Phys. 5, 633 (2009).

[3] H. Weimer, M. Müller, I. Lesanovsky, P. Zoller, and H. P. Büchler, A Rydberg quantum simulator, Nature Phys. 6, 382 (2010).

[4] U. Raitzsch, R. Heidemann, H. Weimer, B. Butscher, P. Kollmann, R. Löw, H. P. Büchler, and T. Pfau, Investigation of dephasing rates in an interacting Rydberg gas, New J. Phys. 11, 055014 (2009).

[5] T. E. Lee, H. Häffner, and M. C. Cross, Antiferromagnetic phase transition in a nonequilibrium lattice of Rydberg atoms, Phys. Rev. A 84, 031402 (2011).

[6] J. Honer, R. Löw, H. Weimer, T. Pfau, and H. P. Büchler, Artificial Atoms Can Do More Than Atoms: Deterministic Single Photon Subtraction from Arbitrary Light Fields, Phys. Rev. Lett. 107, 093601 (2011).

[7] H. Weimer, M. Müller, H. P. Büchler, and I. Lesanovsky, Digital quantum simulation with Rydberg atoms, Quant. Inf. Proc. 10, 885 (2011).

[8] A. W. Glaetzle, R. Nath, B. Zhao, G. Pupillo, and P. Zoller, Driven-dissipative dynamics of a strongly interacting Rydberg gas, Phys. Rev. A 86, 043403 (2012).

[9] C. Ates, B. Olmos, J. P. Garrahan, and I. Lesanovsky, Dynamical phases and intermittency of the dissipative quantum Ising model, Phys. Rev. A 85, 043620 (2012).

[10] M. Lemeshko and H. Weimer, Dissipative binding of atoms by non-conservative forces, Nature Commun. 4, 2230 (2013).

[11] C. Carr, R. Ritter, C. G. Wade, C. S. Adams, and K. J. Weatherill, Nonequilibrium Phase Transition in a Dilute Rydberg Ensemble, Phys. Rev. Lett. 111, 113901 (2013).

[12] D. D. B. Rao and K. Mølmer, Dark Entangled Steady States of Interacting Rydberg Atoms, Phys. Rev. Lett. 111, 033606 (2013).

[13] A. W. Carr and M. Saffman, Preparation of Entan- gled and Antiferromagnetic States by Dissipative Rydberg Pumping, Phys. Rev. Lett. 111, 033607 (2013).

[14] A. Hu, T. E. Lee, and C. W. Clark, Spatial correlations of one-dimensional driven-dissipative systems of Rydberg atoms, Phys. Rev. A 88, 053627 (2013).

[15] M. Höning, D. Muth, D. Petrosyan, and M. Fleischhauer, Steady-state crystallization of Rydberg excitations in an optically driven lattice gas, Phys. Rev. A 87, 023401 (2013).

[16] J. Otterbach and M. Lemeshko, Dissipative Preparation of Spatial Order in Rydberg-Dressed Bose-Einstein Condensates, Phys. Rev. Lett. 113, 070401 (2014).

[17] J. Sanders, R. van Bijnen, E. Vredenbregt, and S. Kokkelmans, Wireless Network Control of Interacting Rydberg Atoms, Phys. Rev. Lett. 112, 163001 (2014).

[18] D. W. Schönleber, M. Gärttner, and J. Evers, Coherent versus incoherent excitation dynamics in dissipative many-body Rydberg systems, Phys. Rev. A 89, 033421 (2014).

[19] N. Malossi, M. M. Valado, S. Scotto, P. Huillery, P. Pillet, D. Ciampini, E. Arimondo, and O. Morsch, Full Counting Statistics and Phase Diagram of a Dissipative Rydberg Gas, Phys. Rev. Lett. 113, 023006 (2014).

[20] M. Hoening, W. Abdussalam, M. Fleischhauer, and T. Pohl, Antiferromagnetic long-range order in dissipative Rydberg lattices, Phys. Rev. A 90, 021603 (2014).

[21] M. Marcuzzi, E. Levi, S. Diehl, J. P. Garrahan, and I. Lesanovsky, Universal Nonequilibrium Properties of Dissipative Rydberg Gases, Phys. Rev. Lett. 113, 210401 (2014).

[22] J. Qian and W. Zhang, Dissipation-sensitive multiphoton excitations of strongly interacting Rydberg atoms, Phys. Rev. A 90, 033406 (2014).

[23] A. Urvoy, F. Ripka, I. Lesanovsky, D. Booth, J. P. Shaffer, T. Pfau, and R. Löw, Strongly correlated growth of Rydberg aggregates in a vapour cell, arXiv:1408.0039 (2014).

[24] T. M. Weber, M. Honing, T. Niederprum, T. Manthey, O. Thomas, V. Guarrera, M. Fleischhauer, G. Barontini, and H. Ott, Mesoscopic Rydberg-blockaded ensembles in 
the superatom regime and beyond, Nature Phys. 11, 157 (2015).

[25] H. Weimer, Variational Principle for Steady States of Dissipative Quantum Many-Body Systems, Phys. Rev. Lett. 114, 040402 (2015).

[26] R. Löw, H. Weimer, J. Nipper, J. B. Balewski, B. Butscher, H. P. Büchler, and T. Pfau, An experimental and theoretical guide to strongly interacting Rydberg gases, J. Phys. B 45, 113001 (2012).

[27] B. Zhao, A. W. Glaetzle, G. Pupillo, and P. Zoller, Atomic Rydberg Reservoirs for Polar Molecules, Phys. Rev. Lett. 108, 193007 (2012).

[28] H.-P. Breuer and F. Petruccione, The Theory of Open Quantum Systems (Oxford University Press, Oxford, 2002).

[29] A. Gilchrist, N. K. Langford, and M. A. Nielsen, Distance measures to compare real and ideal quantum processes, Phys. Rev. A 71, 062310 (2005).

[30] C. V. Kraus and T. J. Osborne, Time-dependent variational principle for dissipative dynamics, Phys. Rev. A 86, 062115 (2012).

[31] B. Edegger, V. N. Muthukumar, and C. Gros, Gutzwiller-RVB theory of high-temperature superconductivity: Results from renormalized mean-field theory and variational Monte Carlo calculations, Adv. Phys. 56, 927 (2007).

[32] R. Liboff, Kinetic Theory: Classical, Quantum, and Rel- ativistic Descriptions (Springer, New York, 2003).

[33] P. Navez and R. Schützhold, Emergence of coherence in the Mott-insulator-superfluid quench of the BoseHubbard model, Phys. Rev. A 82, 063603 (2010).

[34] S. Diehl, W. Yi, A. J. Daley, and P. Zoller, DissipationInduced $d$-Wave Pairing of Fermionic Atoms in an Optical Lattice, Phys. Rev. Lett. 105, 227001 (2010).

[35] J. Johansson, P. Nation, and F. Nori, QuTiP 2: A Python framework for the dynamics of open quantum systems, Comp. Phys. Comm. 184, 1234 (2013).

[36] N. Goldenfeld, Lectures on Phase Transitions and the Renormalization Group (Perseus Books, Reading, 1992).

[37] H. Weimer, R. Löw, T. Pfau, and H. P. Büchler, Quantum critical behavior in strongly interacting Rydberg gases, Phys. Rev. Lett. 101, 250601 (2008).

[38] P. Schauß, M. Cheneau, M. Endres, T. Fukuhara, S. Hild, A. Omran, T. Pohl, C. Gross, S. Kuhr, and I. Bloch, Observation of spatially ordered structures in a twodimensional Rydberg gas, Nature 491, 87 (2012).

[39] R. Heidemann, U. Raitzsch, V. Bendkowsky, B. Butscher, R. Low, and T. Pfau, Rydberg Excitation of BoseEinstein Condensates, Phys. Rev. Lett. 100, 033601 (2008).

[40] P. Zoller, Atomic relaxation and resonance fluorescence in intensity and phase-fluctuating laser light, J. Phys. B 11, 2825 (1978). 